\title{
A Short and Versatile Route to a Key Intermediate for Lactacystin Synthesis
}

\section{Philip C. Bulman Page,* A. Sazali Hamzah, David C. Leach, Steven M. Allin, David M. Andrews, Gerasimos A. Rassias}

\section{Potassium benzyloxycarbonyl acetate}

A solution of $\mathrm{KOH}(9.8 \mathrm{~g}, 0.176 \mathrm{~mol})$ in benzyl alcohol $(176 \mathrm{ml})$ was added gradually to a solution of dibenzyl malonate $(50 \mathrm{~g}, 0.176 \mathrm{~mol})$ in benzyl alcohol $(440 \mathrm{ml})$. The reaction mixture was stirred for four hours at ambient temperature, and diluted with diethyl ether $(2.5 \mathrm{~L})$, resulting in a white precipitate. The mixture was stirred for thirty minutes and the product removed by filtration, washed with diethyl ether $(3 \mathrm{x}$ $200 \mathrm{ml}$ ), and air-dried to give a colourless solid (33.4 g, 82\%); mp $201{ }^{\circ} \mathrm{C}$; (Found: C, 51.59\%; H, 3.75\%. $\mathrm{C}_{10} \mathrm{H}_{9} \mathrm{KO}_{4}$ requires C, 51.71\%; H, 3.91\%); $\square_{\max }(\operatorname{mull}) / \mathrm{cm}^{-1}$ : 1724; (Found: $\mathrm{MH}^{+}, 233.02131 . \mathrm{C}_{10} \mathrm{H}_{9} \mathrm{KO}_{4}$ requires 232.01379); m/z: $233(\mathrm{M}+1,18), 192(100), 154(41), 136(34) ; \square_{\mathrm{H}}\left(\mathrm{D}_{2} \mathrm{O}, 400 \mathrm{MHz}\right): 3.35(2 \mathrm{H}, \mathrm{s})$, $5.16(2 \mathrm{H}, \mathrm{s}), 7.38-7.46(5 \mathrm{H}, \mathrm{m})$; $\mathrm{C}_{\mathrm{c}}\left(\mathrm{D}_{2} \mathrm{O}, 100 \mathrm{MHz}\right): 47.57,70.11,131.08,131.47,131.73,138.44,174.03$, 176.78 .

\section{$N$-Benzylglycine ethyl ester}

Ethyl bromoacetate $(24.4 \mathrm{ml}, 0.22 \mathrm{~mol})$ in THF $(120 \mathrm{ml})$ was added gradually to a solution of benzylamine (42.9 ml, $0.484 \mathrm{~mol})$ in THF $(120 \mathrm{ml})$ at $0{ }^{\circ} \mathrm{C}$, resulting in a white mixture. The mixture was stirred at ambient temperature for four hours, and evaporated to dryness. Diethyl ether $(150 \mathrm{ml})$ was added, the mixture filtered, and the filtrate concentrated to give the product $(40.2 \mathrm{~g}, 95 \%)$. The product was purified for analytical purposes by column chromatography on silica gel using diethyl ether as eluent, but can be used crude. $\square_{\max }\left(\right.$ neat) $/ \mathrm{cm}^{-1}: 3339,1736,1027$; (Found: $\mathrm{M}^{+}, 193.11030 . \mathrm{C}_{11} \mathrm{H}_{15} \mathrm{NO}_{2}$ requires 193.11028); m/z: 193 (M+, 4\%), 120 (54), 106 (30.), 91 (100); $\square_{\mathrm{H}}\left(\mathrm{CDCl}_{3}, 400 \mathrm{MHz}\right): 1.17$ (3H, t, J = 8.0 Hz), 1.86 (1H, br s), 3.30 $(2 \mathrm{H}, \mathrm{s}), 3.70(2 \mathrm{H}, \mathrm{s}), 4.08(2 \mathrm{H}, \mathrm{q}, \mathrm{J}=4.0 \mathrm{~Hz}), 7.14-7.23(5 \mathrm{H}, \mathrm{m}) ; \mathrm{C}_{\mathrm{c}}\left(\mathrm{CDCl}_{3}, 100 \mathrm{MHz}\right): 13.20,49.08,52.25$, $59.64,126.10,127.23,127.40,138.55,171.36$.

\section{$N$-Benzyl- $N$-ethoxycarbonylmethyl malonic acid monoamide benzyl ester 3}

Potassium benzyloxycarbonyl acetate (33 g, $0.142 \mathrm{~mol}$ ) was suspended in toluene (300 ml), and oxalyl chloride $(37.2 \mathrm{ml}, 0.462 \mathrm{~mol})$ added. The reaction was stirred at $34{ }^{\circ} \mathrm{C}$ for twenty-four hours, and the solvent and excess oxalyl chloride removed to give the acid chloride. The crude acid chloride was dissolved in dichloromethane $(200 \mathrm{ml})$, and added to a solution of $N$-benzylglycine ethyl ester (33 $\mathrm{g}, 0.17 \mathrm{~mol})$ in dichloromethane $(200 \mathrm{ml})$ at $0{ }^{\circ} \mathrm{C}$. Pyridine $(13.8 \mathrm{ml}, 0.17 \mathrm{mmol})$ and DMAP $(0.86 \mathrm{~g}, 7.1 \mathrm{mmol})$ were added at $0{ }^{\circ} \mathrm{C}$, the mixture was allowed to reach ambient temperature, and stirred for four hours. The reaction mixture was washed with $5 \% \mathrm{HCl}(2 \times 100 \mathrm{ml})$ and water $(2 \times 100 \mathrm{ml})$, dried $\left(\mathrm{MgSO}_{4}\right)$, and the solvents 
removed to give 3 as an orange oil $(49.8 \mathrm{~g}, 83 \%)$, $\square_{\max }\left(\right.$ neat) $/ \mathrm{cm}^{-1}: 3030,2982,1740,1662,1453,1265$, 1196, 733; (Found: $\mathrm{M}^{+}, 369.15703 . \mathrm{C}_{21} \mathrm{H}_{23} \mathrm{NO}_{5}$ requires 369.15762); m/z: 369 (M+3\%), 278 (7), 192 (99), 118 (12), 91 (100); $\mathrm{\square}_{\mathrm{H}}\left(\mathrm{CDCl}_{3}, 400 \mathrm{MHz}\right): 1.13(3 \mathrm{H}, \mathrm{t}, \mathrm{J}=6.8 \mathrm{~Hz}$, minor rotamer), 1.15 (3H, t, J = 9.2 Hz, major rotamer), 3.45 (2H, s, minor rotamer), 3.52 (2H, s, major rotamer), 3.84 (2H, s, minor rotamer), 3.96 $(2 \mathrm{H}, \mathrm{s}$, major rotamer), $4.04(2 \mathrm{H}, \mathrm{q}, \mathrm{J}=7.2 \mathrm{~Hz}$, minor rotamer), 4.07 ( $2 \mathrm{H}, \mathrm{q}, \mathrm{J}=7.2 \mathrm{~Hz}$, major rotamer), $4.53(2 \mathrm{H}, \mathrm{s}$, major rotamer), 4.59 (2H, s, minor rotamer), 5.08 (2H, s, major rotamer), 5.11 (2H, s, minor rotamer), 6.99-7.27 $(20 \mathrm{H}, \mathrm{m})$; $\mathrm{Z}\left(\mathrm{CDCl}_{3}, 100 \mathrm{MHz}\right): 14.11$ (minor rotamer), 14.14 (major rotamer), 41.07 (major rotamer), 41.34 (minor rotamer), 47.16 (major rotamer), 49.02 (minor rotamer), 49.92 (minor rotamer), 52.79 (major rotamer), 61.26 (major rotamer), 61.78 (minor rotamer), 67.28 (major rotamer), 67.37 (minor rotamer), 126.96-136.08, 166.52 (minor rotamer), 166.90 (major rotamer), 167.03 (major rotamer), 167.21 (minor rotamer), 168.76 (minor rotamer), 168.80 (major rotamer).

\section{$N$-Benzyl-3-methylpyrrolidin-2,4-dione 3-carboxylic acid benzyl ester 9}

Tetrabutylammonium fluoride (174 $\mathrm{ml}$ of a $1 \mathrm{M}$ solution, $174 \mathrm{mmol}$ ) was added to a solution of the diester 3 (32 g, $86.6 \mathrm{mmol}$ ) and in diethyl ether $(250 \mathrm{ml})$, the mixture stirred under $\mathrm{N}_{2}$ at ambient temperature for $c a$ thirty minutes, and the colourless solid removed by filtration and washed with diethyl ether $(2 \times 100 \mathrm{ml})$. The solid was suspended in THF $(250 \mathrm{ml})$ at ambient temperature, and methyl iodide $(10.8 \mathrm{ml}, 173.2 \mathrm{mmol})$ added. The mixture was stirred at ambient temperature for twenty-four hours, and evaporated to dryness. The crude product was purified by column chromatography on silica gel using petroleum ether/ethyl acetate $(7 / 3)$ and ethyl acetate as eluents to give the product 9 as a yellow powder $(15.6 \mathrm{~g}, 53 \%), \mathrm{mp} 62{ }^{\circ} \mathrm{C}$, (Found: C, 70.83\%; H, 5.57\%; N, 3.95\%. $\mathrm{C}_{20} \mathrm{H}_{19} \mathrm{NO}_{4}$ requires C, 71.2\%; H, 5.68\%; N, 4.15\%); $\square_{\max }\left(\mathrm{CH}_{2} \mathrm{Cl}_{2}\right) / \mathrm{cm}^{-1}$ : 1777, 1741, 1685, 1115; (Found: $\mathrm{M}^{+}, 337.13135 . \mathrm{C}_{20} \mathrm{H}_{19} \mathrm{NO}_{4}$ requires 337.13141); m/z: 337 (M+, 7\%), 246 (45), 202 (65), 91 (100); $\square_{\mathrm{H}}\left(\mathrm{CDCl}_{3}, 400 \mathrm{MHz}\right): 1.56(3 \mathrm{H}, \mathrm{s}), 3.66(1 \mathrm{H}, \mathrm{d}, \mathrm{J}=17.6 \mathrm{~Hz}), 3.87$ (1H, d, J = 17.5 Hz), $4.36(1 \mathrm{H}, \mathrm{d}, \mathrm{J}=14.8 \mathrm{~Hz}), 4.93(1 \mathrm{H}, \mathrm{d}, \mathrm{J}=14.8 \mathrm{~Hz}), 5.13(1 \mathrm{H}, \mathrm{d}, \mathrm{J}=12.4 \mathrm{~Hz}), 5.17(1 \mathrm{H}, \mathrm{d}, \mathrm{J}=12.4$ $\mathrm{Hz}), 7.15-7.34(10 \mathrm{H}, \mathrm{m})$; $\mathrm{飞}\left(\mathrm{CDCl}_{3}, 100 \mathrm{MHz}\right): 15.25,46.14,54.95,59.03,68.13,127.76-134.70,165.30$, 169.40, 202.14; and the enol ether by-product as a yellow oil (4.67 g, 16\%), $\square_{\max }\left(\mathrm{CH}_{2} \mathrm{Cl}_{2}\right) / \mathrm{cm}^{-1}: 1718,1618$, 1068; (Found: $\mathrm{M}^{+}, 337.13052 . \mathrm{C}_{20} \mathrm{H}_{19} \mathrm{NO}_{4}$ requires 337.13141); m/z: 337 (M', 2\%), 246 (5), 189 (7), 108 (100), 91 (41); $\mathrm{G}_{\mathrm{H}}\left(\mathrm{CDCl}_{3}, 400 \mathrm{MHz}\right): 3.85(2 \mathrm{H}, \mathrm{s}), 3.89(3 \mathrm{H}, \mathrm{s}), 4.58(2 \mathrm{H}, \mathrm{s}), 5.29(2 \mathrm{H}, \mathrm{s}), 7.20-7.46(10 \mathrm{H}$, m); $\mathrm{Cc}_{\mathrm{c}}\left(\mathrm{CDCl}_{3}, 100 \mathrm{MHz}\right): 45.81,47.70,59.07,66.36,102.10,128.14-137.16,162.21,168.03,177.53$.

\section{$N$-Benzyl-3-methylpyrrolidin-2,4-dione 3-carboxylic acid benzyl ester 9}

Tetrabutylammonium fluoride ( $15 \mathrm{ml}$ of a $1 \mathrm{M}$ solution, $15 \mathrm{mmol}$ ) was added to a solution of the lactam 6 (4 $\mathrm{g}, 12.4 \mathrm{mmol})$ in THF $(140 \mathrm{ml})$, followed by methyl iodide $(1.6 \mathrm{ml}, 24.8 \mathrm{mmol})$. The resulting orange solution was stirred at ambient temperature for twenty-four hours, and the solvent removed to give an orange solid. The crude material was purified by column chromatography on silica gel using petroleum 
ether/ ethyl acetate (6/4) as eluent to give the desired compound 9 as a yellow crystalline solid (2.31 g, $70 \%)$. The spectroscopic data for 9 is given above.

\section{N-Benzyl-3-methylpyrrolidin-2,4-dione 3,5-dicarboxylic acid 3-benzyl ester 5-methyl ester 8}

LHMDS (5.92 ml, $5.92 \mathrm{mmol})$ and DMPU (0.72 $\mathrm{ml}, 5.92 \mathrm{mmol})$ were added to THF $(10 \mathrm{ml})$ under $\mathrm{N}_{2}$, and the mixture cooled to $-78{ }^{\circ} \mathrm{C}$. A solution of lactam $9(1 \mathrm{~g}, 2.96 \mathrm{mmol})$ in THF $(3 \mathrm{ml})$ was added gradually, and the resulting orange solution stirred at $-78^{\circ} \mathrm{C}$ for one hour. Methyl cyanoformate $(0.47 \mathrm{ml}, 5.92 \mathrm{mmol})$ was added, and the solution stirred at $-78{ }^{\circ} \mathrm{C}$ for a further two hours, saturated aqueous $\mathrm{NH}_{4} \mathrm{Cl}(10 \mathrm{ml})$ added, and the mixture diluted with dichloromethane $(50 \mathrm{ml})$. The solution was washed with water $(2 \times 20$ $\mathrm{ml})$ and brine $(2 \times 20 \mathrm{ml})$, dried $\left(\mathrm{MgSO}_{4}\right)$, and the solvents removed. The residue was purified by column chromatography on silica gel using petroleum ether/ethyl acetate (7/3) as eluent to give the product 8 as a 5:1 mixture of inseparable diastereoisomers (0.87 g, 75\%). (Found: C, 66.29\%; H, 5.28\%; N, 3.51\%. $\mathrm{C}_{22} \mathrm{H}_{2}{ }_{1} \mathrm{NO}_{6}$ requires C, 66.83\%; H, 5.35\%; N, 3.54\%); $\square_{\max }$ (neat)/cm ${ }^{-1}: 1789,1749,1707,1218$; (Found $\mathrm{MH}^{+}, 396.14494, \mathrm{C}_{22} \mathrm{H}_{21} \mathrm{NO}_{6} \mathrm{H}^{+}$requires 396.14472); m/z: $395\left(\mathrm{M}^{+}, 2 \%\right), 304$ (22), 260 (37), 105 (76), 91 (100), 77 (97); $\mathrm{G}_{\mathrm{f}}\left(\mathrm{CDCl}_{3}, 400 \mathrm{MHz}\right): 1.56$ (3H, s major isomer), 1.60 (3H, s, minor isomer), 3.46 (3H, s minor isomer), $3.74(3 \mathrm{H}$, s major isomer), $4.00(1 \mathrm{H}, \mathrm{d}, \mathrm{J}=14.8 \mathrm{~Hz}$, major isomer), $4.23(1 \mathrm{H}, \mathrm{d}, \mathrm{J}=14.8 \mathrm{~Hz}$, minor isomer), $4.31(1 \mathrm{H}, \mathrm{s}$, minor isomer), $4.48(1 \mathrm{H}, \mathrm{s}$, major isomer), $5.04(1 \mathrm{H}, \mathrm{d}, \mathrm{J}=12.4 \mathrm{~Hz}$, minor isomer), $5.08(1 \mathrm{H}, \mathrm{d}, \mathrm{J}=12.4 \mathrm{~Hz}$, major isomer), $5.19(1 \mathrm{H}, \mathrm{d}, \mathrm{J}=12.4 \mathrm{~Hz}$, major isomer), $5.22(1 \mathrm{H}, \mathrm{d}, \mathrm{J}=$ $12.4 \mathrm{~Hz}$, minor isomer), $5.35(1 \mathrm{H}, \mathrm{d}, \mathrm{J}=14.8 \mathrm{~Hz}$, major isomer), $5.39(1 \mathrm{H}, \mathrm{d}, \mathrm{J}=14.8 \mathrm{~Hz}$, minor isomer), 7.09-7.35 (20H, m); $\left(\mathrm{CDCl}_{3}, 100 \mathrm{MHz}\right): 15.96,16.03,45.15$ (major isomer), 45.36 (minor isomer), 52.84 (minor isomer), 53.23 (major isomer), 58.14 (minor isomer), 58.42 (major isomer), 66.69 (minor isomer), 67.53 (major isomer), 68.14 (minor isomer), 68.30 (major isomer), 126.9-134.5, 163.73, 164.70, 164.80, $164.95,167.65,168.81,195.78$ (minor isomer), 196.85 (major isomer).

\section{$\mathrm{N}$-Benzyl-3-methylpyrrolidin-2,4-dione 5-carboxylic acid methyl ester 14}

Keto-ester $8(300 \mathrm{mg}, 0.76 \mathrm{mmol})$ and $\mathrm{Pd}(\mathrm{OH})_{2} / \mathrm{C}(30 \mathrm{mg})$ were added to THF $(15 \mathrm{ml})$ and the mixture degassed with nitrogen. The mixture was treated with hydrogen under balloon pressure for 20 minutes at room temperature, filtered through celite and evaporated to dryness to give the title compound $\mathbf{1 4}$ as a colourless solid (188 mg, 95\%), mp 123-124 ${ }^{\circ} \mathrm{C}$, $\square_{\max }(\operatorname{mull}) / \mathrm{cm}^{-1}$ : 3038, 1746, 1212; (Found: $\mathrm{M}^{+}$, 261.10021. $\mathrm{C}_{14} \mathrm{H}_{15} \mathrm{NO}_{4}$ requires 261.10011); $\mathrm{m} / \mathrm{z}:\left(\mathrm{M}^{+}, 15 \%\right), 202$ (21), 91 (100), 65 (10); $\square_{\mathrm{H}}\left(\mathrm{CDCl}_{3}\right.$, 400MHz): $1.35(3 \mathrm{H}, \mathrm{d}, \mathrm{J}=7.6 \mathrm{~Hz}$, major isomer), $1.42(3 \mathrm{H}, \mathrm{d}, \mathrm{J}=7.6 \mathrm{~Hz}$, minor isomer $), 2.95(1 \mathrm{H}, \mathrm{q}, \mathrm{J}=$ $7.6 \mathrm{~Hz}$, minor isomer), $3.11(1 \mathrm{H}, \mathrm{q}, \mathrm{J}=7.6 \mathrm{~Hz}$, major isomer), 3.71 (3H, s, major isomer), $3.74(3 \mathrm{H}, \mathrm{s}$, minor isomer), $4.07(1 \mathrm{H}, \mathrm{d}, \mathrm{J}=14.4 \mathrm{~Hz}$, minor isomer), $4.19(1 \mathrm{H}, \mathrm{d}, \mathrm{J}=14.6 \mathrm{~Hz}$, major isomer), $4.28(1 \mathrm{H}, \mathrm{s}$, major), 4.40 (1H, s, minor isomer), $5.18(1 \mathrm{H}, \mathrm{d}, \mathrm{J}=14.8 \mathrm{~Hz}$, major isomer), $5.28(1 \mathrm{H}, \mathrm{d}, \mathrm{J}=14.8 \mathrm{~Hz}$, minor isomer), 7.21-7.35 (10H, m); $\square c\left(\mathrm{CDCl}_{3}, 100 \mathrm{MHz}\right)$ : 9.76 (major isomer), 11.98 (minor isomer), 44.43 (major isomer), 44.65 (minor isomer), 45.16 (minor isomer), 15.28 (major isomer), 53.38, 67.91, 127.64-1129.50, 
134.44, 165.34 (major isomer), 165.49 (minor isomer), 171.88 (major isomer), 172.26 (minor isomer), 200.29 (major isomer), 201.26 (minor isomer). 